\title{
TALBOT EFFECT FOR THE CUBIC NON-LINEAR SCHRÖDINGER EQUATION ON THE TORUS
}

\author{
M.B. ERDOĞAN AND N. TZIRAKIS
}

\begin{abstract}
We study the evolution of the one dimensional periodic cubic Schrödinger equation (NLS) with bounded variation data. For the linear evolution, it is known that for irrational times the solution is a continuous, nowhere differentiable fractal-like curve. For rational times the solution is a linear combination of finitely many translates of the initial data. Such a dichotomy was first observed by Talbot in an optical experiment performed in 1836, [20]. In this paper, we prove that a similar phenomenon occurs in the case of the NLS equation.
\end{abstract}

\section{Introduction}

In a 1836 optical experiment, Talbot [20] observed white light passing through a diffraction grating. He looked at the images that were produced with the help of magnifying lens and noticed a sharp focused pattern with a certain periodicity depending on the distance. Rayleigh [18] calculated the Talbot distance as $d=\frac{\alpha^{2}}{\lambda}$, where $\alpha$ is the spacing of the grating and $\lambda$ is the wavelength of the incoming light.

Berry with his collaborators (see, e.g., [1-4]) studied the Talbot effect in a series of papers. In particular, Berry and Klein [2] used the linear Schrödinger evolution to model the Talbot effect. They showed that at rational multiples of the Talbot distance overlapping copies of the grating pattern reappear, while at irrational times the images have a fractal nowhere differentiable profile. Also Berry [1] conjectured that for the $n$-dimensional linear Schrödinger equation confined in a box the imaginary part $\Im u(x, t)$, the real part $\Re u(x, t)$ and the density $|u(x, t)|^{2}$ of the solution is a fractal set with dimension $D=n+\frac{1}{2}$ for most irrational times. He also observed that in the one dimensional case there are space slices whose time fractal dimension is $\frac{7}{4}$ and there are diagonal slices with dimension $\frac{5}{4}$.

The first mathematically rigorous work in this area appears to be due to Oskolkov. Oskolkov [17] studied a large class of linear dispersive equations with bounded variation initial data. In the case of the linear Schrödinger equation, he proved that at rational times the solution is a continuous function of $x$ and at rational times it is a bounded function with at most countably many discontinuities. For the exact statement see Theorem 3 below.

The idea that the profile of linear dispersive equations depend on the algebraic properties of time have been further exploited in the papers of Kapitanski and Rodniaski [14], Rodnianski [19], and Taylor [21]. Kapitanski and Rodnianski [14] showed that the solution to the linear Schrödinger equation has better regularity properties (measured in Besov spaces) at irrational than rational times. It is important to note

Received by the editors March 11, 2013. 
that this subtle effect cannot be observed in the scale of Sobolev spaces since the linear propagator is a unitary operator in Sobolev spaces. In [19], using the result in [14], Rodnianski partially justified Berry's conjecture in one dimension, see Section 2.

Taylor [21] independently obtained Berry's quantization result and also extended it to higher dimensional spheres and tori [22]. In particular he proved that at rational times the solution is a linear combination of finitely many translates of the initial data with the coefficients being Gauss sums. He further showed that some classical identities for Gauss sums can be obtained by an analysis of the linear Schrödinger evolution. This should not come as a surprise since number theoretic connections of Talbot effect had already been observed in the works of Oskolkov, Kapitanski and Rodnianski. Taylor [21] also noted that the quantization implies the $L^{p}$ boundedness of the multiplier $e^{i t \Delta}$ for rational values of $\frac{t}{2 \pi}$. It is known that, Taylor [21], the propagator is unbounded in $L^{p}$ for $p \neq 2$ and $\frac{t}{2 \pi}$ irrational. This can be considered as another manifestation of the Talbot effect.

More recently Olver [16], and Chen and Olver [8,9] provided numerical simulations of the Talbot effect for a large class of dispersive equations, both linear and non-linear. In the case of polynomial dispersion, they numerically confirmed the rational/irrational dichotomy discussed above. This behavior persists for both integrable and non-integrable systems. An important question that the authors raised is the appearance of such phenomena in the case of non-polynomial dispersion relations. The numerics demonstrate that the large wave number asymptotics of the dispersion relation plays the dominant role governing the qualitative features of the solutions. We should also note that in [24] the Talbot effect was observed experimentally in a non-linear setting.

In this note, we investigate the Talbot effect for cubic non-linear Schrödinger (NLS) equation with periodic boundary conditions. Our goal is to extend Oskolkov's and Rodnianski's results for bounded variation data to the NLS evolution, and provide rigorous confirmation of some numerical observations in $[8,9,16]$. In particular we prove that for a large class of rough data the solution of the NLS equation, for almost all times, is a continuous but fractal-like curve with upper Minkowski dimension ${ }^{1} \frac{3}{2}$. Our main result is the following theorem:

Theorem 1. Consider the NLS equation on the torus:

$$
\begin{aligned}
& i u_{t}+u_{x x}+|u|^{2} u=0, \quad t \in \mathbb{R}, \quad x \in \mathbb{T}=\mathbb{R} / 2 \pi \mathbb{Z}, \\
& u(x, 0)=g(x) .
\end{aligned}
$$

Assuming that $g$ is of bounded variation, we have

(i) $u(x, t)$ is a continuous function of $x$ if $\frac{t}{2 \pi}$ is an irrational number. For rational values of $\frac{t}{2 \pi}$, the solution is a bounded function with at most countably many discontinuities. Moreover, if $g$ is also continuous then $u \in C_{t}^{0} C_{x}^{0}$.

\footnotetext{
${ }^{1}$ Upper Minkowski (also known as fractal) dimension, $\overline{\operatorname{dim}}(E)$, of a bounded set $E$ is given by

$$
\limsup _{\epsilon \rightarrow 0} \frac{\log (\mathcal{N}(E, \epsilon))}{\log \left(\frac{1}{\epsilon}\right)},
$$
}

where $\mathcal{N}(E, \epsilon)$ is the minimum number of $\epsilon$-balls required to cover $E$. 
(ii) If in addition $g \notin \bigcup_{\epsilon>0} H^{\frac{1}{2}+\epsilon}$, then for almost all times either the real part or the imaginary part of the graph of $u(\cdot, t)$ has upper Minkowski dimension $\frac{3}{2}$.

We note that the simulations in $[8,9,16]$ were performed in the case when $g$ is a step function, and that Theorem 1 applies in that particular case.

To prove Theorem 1 we first obtain a smoothing result for NLS stating that the non-linear Duhamel part of the evolution is smoother than the linear part by almost half a derivative. For bounded variation data, this implies that the non-linear part is in $H^{1-}$ which immediately yields the upper bound on the dimension of the curve. The lower bound is obtained by combining our smoothing estimate with Rodnianski's result in [19], and an observation from Deliu and Jawerth [11] connecting smoothness and geometric dimension. We remark that the first part of Theorem 1 was observed in [12] in the case of Korteweg-de Vries (KdV) equation.

\section{Discussion of earlier results and the proof of Theorem 1}

First of all recall that for $s \geq 0, H^{s}(\mathbb{T})$ is defined as a subspace of $L^{2}$ via the norm

$$
\|f\|_{H^{s}(\mathbb{T})}:=\sqrt{\sum_{k \in \mathbb{Z}}\langle k\rangle^{2 s}|\widehat{f}(k)|^{2}},
$$

where $\langle k\rangle:=\left(1+k^{2}\right)^{1 / 2}$ and $\widehat{f}(k)=\int_{0}^{2 \pi} f(x) e^{-i k x} d x$ are the Fourier coefficients of $f$. We note that the local and global wellposedness of NLS for $H^{s}$ data for $s \geq 0$ was obtained by Bourgain [5].

We start by formally decomposing the solution as

$$
u(x, t)=e^{i\left(\partial_{x x}+P\right) t} g+\mathcal{N}(x, t),
$$

where $P=\|g\|_{2}^{2} / \pi$. Here $\mathcal{N}$ is the non-resonant part of the non-linear Duhamel term of the solution. The proof of Theorem 1 relies on earlier results by Oskolkov and by Rodnianski on the linear part $e^{i\left(\partial_{x x}+P\right) t} g$ and the following smoothing theorem involving $\mathcal{N}$.

Theorem 2. Fix $s>0$. Assume that $g \in H^{s}(\mathbb{T})$. Then for any $a<\min (2 s, 1 / 2)$, we have

$$
\mathcal{N}(x, t) \in C_{t \in \mathbb{R}}^{0} H_{x \in \mathbb{T}}^{s+a} .
$$

We note that this type of smoothing does not hold for $s \leq 0$. For this and (local) smoothing results in $\mathcal{F} \ell^{p}$ spaces, see [10]. We will prove Theorem 2 in Section 3 below. Note that if $g$ is of bounded variation then $g \in \bigcap_{\epsilon>0} H^{\frac{1}{2}-\epsilon}$, and hence

$$
\mathcal{N}(x, t) \in \bigcap_{\epsilon>0} C_{t \in \mathbb{R}}^{0} H_{x \in \mathbb{T}}^{1-\epsilon}
$$

In particular, $\mathcal{N}(x, t) \in C_{t}^{0} C_{x}^{0}$. This and the following theorem ${ }^{2}$ of Oskolkov [17] conclude the proof of part (i) of Theorem 1 .

\footnotetext{
${ }^{2}$ In fact Theorem 3 is a special case of a theorem of Oskolkov which asserts the statement above for one-dimensional dispersive equations with polynomial dispersion relation.
} 
Theorem 3 (Oskolkov [17]). Let $g$ be of bounded variation, then $e^{i t \partial_{x x}} g$ is a continuous function of $x$ if $\frac{t}{2 \pi}$ is an irrational number. For rational values of $\frac{t}{2 \pi}$, it is a bounded function with at most countably many discontinuities. Moreover, if $g$ is also continuous then $e^{i t \partial_{x x}} g \in C_{t}^{0} C_{x}^{0}$.

Remark 1. In the case when the initial data has jump discontinuities, by the quantization result in $[2,16,21]$, the linear solution has jump discontinuities when $\frac{t}{2 \pi}$ is a rational number, since it is a linear combination of finitely many translates of the initial data. Noting that $\mathcal{N}$ is a continuous function of $x$, we have the same conclusion for the NLS evolution.

The second part of the theorem will rely on a result of Rodnianski [19] which in turn relies on results obtained by Kapitanski and Rodnianski [14]. To state this result we need to define the Besov space $B_{p, \infty}^{s}$ via the norm:

$$
\|f\|_{B_{p, \infty}^{s}}:=\sup _{j \geq 0} 2^{s j}\left\|P_{j} f\right\|_{L^{p}}
$$

where $P_{j}$ is a Littlewood-Paley projection on to the frequencies $\approx 2^{j}$. We should note that $C^{\alpha}(\mathbb{T})$ coincides with $B_{\infty, \infty}^{\alpha}$, see, e.g., [23].

Theorem 4 (Rodnianski [19]). Assume that $g$ is of bounded variation and that $g \notin \bigcup_{\epsilon>0} H^{\frac{1}{2}+\epsilon}$, then for almost all irrational $\frac{t}{2 \pi}$,

$$
\begin{aligned}
& e^{i t \partial_{x x}} g \notin \bigcup_{\epsilon>0} B_{1, \infty}^{\frac{1}{2}+\epsilon}, \\
& e^{i t \partial_{x x}} g \in \bigcap_{\epsilon>0} B_{\infty, \infty}^{\frac{1}{2}-\epsilon}=\bigcap_{\epsilon>0} C^{\frac{1}{2}-\epsilon} .
\end{aligned}
$$

We note that for each $\alpha, H^{\alpha} \in B_{1, \infty}^{\alpha}$, and that, for $0<\alpha<\frac{1}{2}, H^{\alpha+\frac{1}{2}} \subset C^{\alpha}$. Therefore, by Theorem 1, we have for each $t$,

$$
\begin{aligned}
& \mathcal{N}(\cdot, t) \in \bigcap_{\epsilon>0} B_{1, \infty}^{1-\epsilon} \\
& \mathcal{N}(\cdot, t) \in \bigcap_{\epsilon>0} C^{\frac{1}{2}-\epsilon}
\end{aligned}
$$

This implies that Theorem 4 is valid for the non-linear solution $u$.

It is a well-known result that if $f: \mathbb{T} \rightarrow \mathbb{R}$ is in $C^{\alpha}$, then the graph of $f$ has upper Minkowski dimension $D \leq 2-\alpha$. Therefore, the graphs of $\Re(u)$ and $\Im(u)$ have dimension at most $\frac{3}{2}$. We note that this upper bound would not have followed if we had less than half a derivative gain in Theorem 2 .

The lower bound follows from the claim of Theorem 4 for the non-linear solution $u$ and the following theorem of Deliu and Jawerth [11].

Theorem 5 (Deliu and Jawerth [11]). The graph of a continuous function $f$ : $\mathbb{T} \rightarrow \mathbb{R}$ has upper Minkowski dimension $D \geq 2-s$ provided that $f \notin \bigcup_{\epsilon>0} B_{1, \infty}^{s+\epsilon}$. 


\section{Proof of Theorem 2}

The theorem follows from a simple $X^{s, b}$ space estimate that appears to be new.

With the change of variable $u(x, t) \rightarrow u(x, t) e^{i P t}$, where $P=\|g\|_{2}^{2} / \pi$, we obtain the equation

$$
i u_{t}+u_{x x}+|u|^{2} u-P u=0, \quad t \in \mathbb{R}, \quad x \in \mathbb{T},
$$

with initial data in $g \in H^{s}, s>0$. We want to prove that for any $a<\min \left(2 s, \frac{1}{2}\right)$, we have

$$
u-e^{i \partial_{x x} t} g \in C_{t \in \mathbb{R}}^{0} H_{x \in \mathbb{T}}^{s+a} .
$$

Note the following identity which follows from Plancherel's theorem:

$$
\begin{aligned}
\widehat{|u|^{2} u}(k)= & \sum_{k_{1}, k_{2}} \widehat{u}\left(k_{1}\right) \overline{\widehat{u}\left(k_{2}\right)} \widehat{u}\left(k-k_{1}+k_{2}\right) \\
= & \frac{1}{\pi}\|u\|_{2}^{2} \widehat{u}(k)-|\widehat{u}(k)|^{2} \widehat{u}(k)+\sum_{k_{1} \neq k, k_{2} \neq k_{1}} \widehat{u}\left(k_{1}\right) \overline{\widehat{u}\left(k_{2}\right)} \widehat{u}\left(k-k_{1}+k_{2}\right) \\
& =: P \widehat{u}(k)+\widehat{\rho(u)}(k)+\widehat{R(u)}(k),
\end{aligned}
$$

where $\widehat{u}(k)=\int_{0}^{2 \pi} u(x) e^{-i k x} d x$ are the Fourier coefficients. Using this in the Duhamel's formula, we have

$$
u(t)=e^{i t \partial_{x x}} g+i \int_{0}^{t} e^{i(t-\tau) \partial_{x x}}(\rho(u)+R(u)) d \tau .
$$

We note that

$$
\|\rho(u)\|_{H^{s+a}}=\sqrt{\sum_{k}|\widehat{u}(k)|^{6}\langle k\rangle^{2 s+2 a}} \lesssim\|u\|_{H^{s}}^{3},
$$

for $0 \leq a \leq 2 s$.

Using (3.1), we have

$$
\left\|u(t)-e^{i t \partial_{x x}} g\right\|_{H^{s+a}} \lesssim \int_{0}^{t}\|u(\tau)\|_{H^{s}}^{3} d \tau+\left\|\int_{0}^{t} e^{i(t-\tau) \partial_{x x}} R(u) d \tau\right\|_{H^{s+a}} .
$$

To bound the integral involving $R(u)$, we work with the $X^{s, b}$ space $[5,6]$ :

$$
\|u\|_{X^{s, b}}=\left\|\widehat{u}(\tau, k)\langle k\rangle^{s}\left\langle\tau-k^{2}\right\rangle^{b}\right\|_{L_{\tau}^{2} \ell_{k}^{2}},
$$

with $b>1 / 2$. We also define the restricted norm

$$
\|u\|_{X_{\delta}^{s, b}}=\inf _{\tilde{u}=u \text { on }[-\delta, \delta]}\|\tilde{u}\|_{X^{s, b}} .
$$

We will use the embedding $X^{s, b} \subset C_{t}^{0} H_{x}^{s}$ for $b>\frac{1}{2}$ and the following inequality from Bourgain [5]. For any $s \in \mathbb{R}, \delta \leq 1$, and $b>\frac{1}{2}$, we have

$$
\left\|\int_{0}^{t} e^{-i(t-s) \partial_{x x}} F(s) d s\right\|_{X_{\delta}^{s, b}} \lesssim\|F\|_{X_{\delta}^{s, b-1}} .
$$

Thus, for $0 \leq t \leq \delta$, we have

$$
\left\|\int_{0}^{t} e^{i(t-\tau) \partial_{x x}} R(u) d \tau\right\|_{H^{s+a}} \lesssim\left\|\int_{0}^{t} e^{i(t-\tau) \partial_{x x}} R(u) d \tau\right\|_{X_{\delta}^{s+a, b}} \lesssim\|R(u)\|_{X_{\delta}^{s+a, b-1}} .
$$

We will prove the following proposition in the next section. 
Proposition 1. For fixed $s>0$ and $a<\min \left(2 s, \frac{1}{2}\right)$, we have

$$
\|R(u)\|_{X^{s+a, b-1}} \lesssim\|u\|_{X^{s, b}}^{3}
$$

provided that $0<b-\frac{1}{2}$ is sufficiently small. The same inequality holds for the restricted norms.

Using Proposition 1, we have for $0<t<\delta<1$ (where $[0, \delta]$ is the local existence interval)

$$
\left\|u(t)-e^{i t \partial_{x x}} g\right\|_{H^{s+a}} \lesssim \int_{0}^{t}\|u(\tau)\|_{H^{s}}^{3} d \tau+\|u\|_{X_{\delta}^{s, b}}^{3} \lesssim\|u\|_{X_{\delta}^{s, b}}^{3} \lesssim\|g\|_{H^{s}}^{3} .
$$

We note that (see, page 113-114 in [7]) the local existence time $\delta$ depends on the $L^{2}$ norm of the data, $\delta=\delta\left(\|g\|_{2}\right)<1$, and by iteration we also have for any $s \geq 0$,

$$
\|u\|_{H^{s}} \leq C e^{C|t|}\|g\|_{H^{s}}=: T(t) .
$$

Fix $t$ large. For $r \leq t$, we have the bound

$$
\|u(r)\|_{H^{s}} \lesssim T(r) \leq T(t)
$$

Using (3.2) repeatedly, we have

$$
\left\|u(j \delta)-e^{i \delta \partial_{x x}} u((j-1) \delta)\right\|_{H^{s+a}} \lesssim\|u((j-1) \delta)\|_{H^{s}}^{3} \lesssim T(t)^{3},
$$

for any $j \leq t / \delta$. Using this we obtain (with $J=t / \delta$ )

$$
\begin{aligned}
\left\|u(J \delta)-e^{i J \delta \partial_{x x}} f\right\|_{H^{s+a}} & \leq \sum_{j=1}^{J}\left\|e^{i(J-j) \delta \partial_{x x}} u(j \delta)-e^{i(J-j+1) \delta \partial_{x x}} u((j-1) \delta)\right\|_{H^{s+a}} \\
& =\sum_{j=1}^{J}\left\|u(j \delta)-e^{i \delta \partial_{x x}} u((j-1) \delta)\right\|_{H^{s+a}} \lesssim J T(t)^{3} \approx t T(t)^{3} / \delta .
\end{aligned}
$$

This finishes the proof of Theorem 2 .

\section{Proof of Proposition 1}

We start with the following elementary lemma (see, e.g., the Appendix of [13]).

Lemma 1. If $\beta \geq \gamma \geq 0$ and $\beta+\gamma>1$, then

$$
\sum_{n} \frac{1}{\left\langle n-k_{1}\right\rangle^{\beta}\left\langle n-k_{2}\right\rangle^{\gamma}} \lesssim\left\langle k_{1}-k_{2}\right\rangle^{-\gamma} \phi_{\beta}\left(k_{1}-k_{2}\right),
$$

and

$$
\int_{\mathbb{R}} \frac{1}{\left\langle\tau-k_{1}\right\rangle^{\beta}\left\langle\tau-k_{2}\right\rangle \gamma} d \tau \lesssim\left\langle k_{1}-k_{2}\right\rangle^{-\gamma} \phi_{\beta}\left(k_{1}-k_{2}\right)
$$

where

$$
\phi_{\beta}(k):=\sum_{|n| \leq|k|} \frac{1}{\langle n\rangle^{\beta}} \sim \begin{cases}1, & \beta>1 \\ \log (1+\langle k\rangle), & \beta=1 \\ \langle k\rangle^{1-\beta}, & \beta<1\end{cases}
$$


Proof of Proposition 1. Using the definition of $X^{s, b}$ norm, we have

$$
\begin{aligned}
& \|R(u)\|_{X^{s+a, b-1}}^{2}= \\
& \left\|\int_{\tau_{1}, \tau_{2}} \sum_{k_{1} \neq k, k_{2} \neq k_{1}} \frac{\langle k\rangle^{s+a} \widehat{u}\left(k_{1}, \tau_{1}\right) \overline{\widehat{u}\left(k_{2}, \tau_{2}\right)} \widehat{u}\left(k-k_{1}+k_{2}, \tau-\tau_{1}+\tau_{2}\right)}{\left\langle\tau-k^{2}\right\rangle^{1-b}}\right\|_{\ell_{k}^{2} L_{\tau}^{2}}^{2} .
\end{aligned}
$$

Let

$$
f(k, \tau)=|\widehat{u}(k, \tau)|\langle k\rangle^{s}\left\langle\tau-k^{2}\right\rangle^{b} .
$$

It suffices to prove that

$$
\begin{aligned}
& \| \int_{\tau_{1}, \tau_{2}} \sum_{k_{1} \neq k, k_{2} \neq k_{1}} M\left(k_{1}, k_{2}, k, \tau_{1}, \tau_{2}, \tau\right) f\left(k_{1}, \tau_{1}\right) f\left(k_{2}, \tau_{2}\right) \\
& \times f\left(k-k_{1}+k_{2}, \tau-\tau_{1}+\tau_{2}\right) \|_{\ell_{k}^{2} L_{\tau}^{2}}^{2} \\
& \lesssim\|f\|_{2}^{6}=\|u\|_{X^{s, b}}^{6},
\end{aligned}
$$

where

$$
\begin{aligned}
& M\left(k_{1}, k_{2}, k, \tau_{1}, \tau_{2}, \tau\right)= \\
& \quad \frac{\langle k\rangle^{s+a}\left\langle k_{1}\right\rangle^{-s}\left\langle k_{2}\right\rangle^{-s}\left\langle k-k_{1}+k_{2}\right\rangle^{-s}}{\left\langle\tau-k^{2}\right\rangle^{1-b}\left\langle\tau_{1}-k_{1}^{2}\right\rangle^{b}\left\langle\tau_{2}-k_{2}^{2}\right\rangle^{b}\left\langle\tau-\tau_{1}+\tau_{2}-\left(k-k_{1}+k_{2}\right)^{2}\right\rangle^{b}} .
\end{aligned}
$$

By Cauchy-Schwarz in $\tau_{1}, \tau_{2}, k_{1}, k_{2}$ variables, we estimate the norm above by

$$
\begin{aligned}
& \sup _{k, \tau}\left(\int_{\tau_{1}, \tau_{2}} \sum_{k_{1} \neq k, k_{2} \neq k_{1}} M^{2}\left(k_{1}, k_{2}, k, \tau_{1}, \tau_{2}, \tau\right)\right) \\
& \quad \times\left\|\int_{\tau_{1}, \tau_{2}} \sum_{k_{1}, k_{2}} f^{2}\left(k_{1}, \tau_{1}\right) f^{2}\left(k_{2}, \tau_{2}\right) f^{2}\left(k-k_{1}+k_{2}, \tau-\tau_{1}+\tau_{2}\right)\right\|_{\ell_{k}^{1} L_{\tau}^{1}} .
\end{aligned}
$$

Note that the norm above is equal to $\left\|f^{2} * f^{2} * f^{2}\right\|_{\ell_{k}^{1} L_{\tau}^{1}}$, which can be estimated by $\|f\|_{2}^{6}$ by Young's inequality. Therefore, it suffices to prove that the supremum above is finite.

Using the second assertion of Lemma 1 in $\tau_{1}$ and $\tau_{2}$ integrals, we obtain

$$
\begin{aligned}
\sup _{k, \tau} \int_{\tau_{1}, \tau_{2}} \sum_{k_{1} \neq k, k_{2} \neq k_{1}} M^{2} & \lesssim \sup _{k, \tau} \sum_{k_{1} \neq k, k_{2} \neq k_{1}} \frac{\langle k\rangle^{2 s+2 a}\left\langle k_{1}\right\rangle^{-2 s}\left\langle k_{2}\right\rangle^{-2 s}\left\langle k-k_{1}+k_{2}\right\rangle^{-2 s}}{\left\langle\tau-k^{2}\right\rangle^{2-2 b}\left\langle\tau-k_{1}^{2}+k_{2}^{2}-\left(k-k_{1}+k_{2}\right)^{2}\right\rangle^{2 b}} \\
& \lesssim \sup _{k} \sum_{k_{1} \neq k, k_{2} \neq k_{1}} \frac{\langle k\rangle^{2 s+2 a}\left\langle k_{1}\right\rangle^{-2 s}\left\langle k_{2}\right\rangle^{-2 s}\left\langle k-k_{1}+k_{2}\right\rangle^{-2 s}}{\left\langle k^{2}-k_{1}^{2}+k_{2}^{2}-\left(k-k_{1}+k_{2}\right)^{2}\right\rangle^{2-2 b}} .
\end{aligned}
$$

The last line follows by the simple fact

$$
\langle\tau-n\rangle\langle\tau-m\rangle \gtrsim\langle n-m\rangle .
$$

Since we have only the non-resonant terms, it suffices to estimate

$$
\sum_{k_{1}, k_{2}} \frac{\langle k\rangle^{2 s+2 a}\left\langle k_{1}\right\rangle^{-2 s}\left\langle k_{2}\right\rangle^{-2 s}\left\langle k-k_{1}+k_{2}\right\rangle^{-2 s}}{\left\langle k-k_{1}\right\rangle^{2-2 b}\left\langle k_{1}-k_{2}\right\rangle^{2-2 b}} .
$$


To estimate this sum we consider the cases $\left|k-k_{1}+k_{2}\right| \gtrsim|k|,\left|k_{1}\right| \gtrsim|k|$, and $\left|k_{2}\right| \gtrsim|k|$. In the estimate, we always take $0<b-\frac{1}{2}$ sufficiently small for any given $s>0$ and $0 \leq a<\min \left(2 s, \frac{1}{2}\right)$.

In the first case, using Lemma 1 we bound the sum by

$$
\sum_{k_{1}, k_{2}} \frac{\langle k\rangle^{2 a}\left\langle k_{1}\right\rangle^{-2 s}\left\langle k_{2}\right\rangle^{-2 s}}{\left\langle k-k_{1}\right\rangle^{2-2 b}\left\langle k_{1}-k_{2}\right\rangle^{2-2 b}} \lesssim \sum_{k_{1}} \frac{\langle k\rangle^{2 a} \phi_{\max (2 s, 2-2 b)}\left(k_{1}\right)}{\left\langle k-k_{1}\right\rangle^{2-2 b}\left\langle k_{1}\right\rangle^{2 s+\min (2-2 b, 2 s)}} .
$$

In the case $s \geq \frac{1}{2}$, we bound the sum by

$$
\sum_{k_{1}} \frac{\langle k\rangle^{2 a} \log \left(1+\left\langle k_{1}\right\rangle\right)}{\left\langle k-k_{1}\right\rangle^{2-2 b}\left\langle k_{1}\right\rangle^{2 s+2-2 b}} \lesssim\langle k\rangle^{2 a-2+2 b+} \lesssim 1
$$

provided $a<\frac{1}{2}$.

In the case $0<s<\frac{1}{2}$, using Lemma 1 we bound the sum by

$$
\sum_{k_{1}} \frac{\langle k\rangle^{2 a}}{\left\langle k-k_{1}\right\rangle^{2-2 b}\left\langle k_{1}\right\rangle^{4 s+1-2 b}} \lesssim \begin{cases}\langle k\rangle^{2 a+4 b-4 s-2} & 0<s \leq \frac{1}{4} \\ \langle k\rangle^{2 a+2 b-2} & \frac{1}{4}<s<\frac{1}{2}\end{cases}
$$

This is bounded in $k$ provided that $0 \leq a<\min \left(2 s, \frac{1}{2}\right)$.

The second case is identical to the first case after renaming the variables: $n_{1}=$ $k-k_{1}+k_{2}, n_{2}=k_{2}$.

In the third case, after renaming the variables $n_{1}=k_{1}, n_{2}=k-k_{1}+k_{2}$, and using Lemma 1 we bound the sum by

$$
\sum_{n_{1}, n_{2}} \frac{\langle k\rangle^{2 a}\left\langle n_{1}\right\rangle^{-2 s}\left\langle n_{2}\right\rangle^{-2 s}}{\left\langle k-n_{1}\right\rangle^{2-2 b}\left\langle k-n_{2}\right\rangle^{2-2 b}} \lesssim\langle k\rangle^{2 a} \phi_{\max (2 s, 2-2 b)}^{2}(k)\langle k\rangle^{-2 \min (2-2 b, 2 s)} \lesssim 1,
$$

provided that $s>0$ and $0 \leq a<\min (2 s, 1)$. This finishes the proof of Proposition 1 .

Remark 2. A similar smoothing property was established in [12] for the $\mathrm{KdV}$ equation. However, in that case, smoothing is not immediate within the context of the $X^{s, b}$ norms as the following example shows [15]. Fix $s \in \mathbb{R}$. Consider $\widehat{u}(n, \tau)=\delta(n-M) \chi_{[-1,1]}\left(\tau-n^{3}\right)$, and $\widehat{v}(n, \tau)=\delta(n-1) \chi_{[-1,1]}\left(\tau-n^{3}\right)$ with $M \gg 1$. The inequality

fails for any $a>0$.

$$
\left\|(u v)_{x}\right\|_{X_{K d V}^{s+a,-1 / 2}} \lesssim\|u\|_{X_{K d V}^{s, 1 / 2}}\|v\|_{X_{K d V}^{s, 1 / 2}}
$$

The analogue of Theorem 2 was proved in [12] after transforming the KdV equation using differentiation by parts. However, the dimension statement in Theorem 1 is open for $\mathrm{KdV}$ since the corresponding result for the linear part (Airy equation) is not known. We will address this issue in future work.

Remark 3. The gain of almost half a derivative in Theorem 2 can be inferred by taking the first Picard iteration for the cubic NLS and test the term

$$
e^{i t \partial_{x x}} \int_{0}^{t} e^{-i t^{\prime} \partial_{x x}}\left[e^{i t^{\prime} \partial_{x x}} g \overline{\left(e^{i t^{\prime} \partial_{x x}} g\right)} e^{i t^{\prime} \partial_{x x}} g\right] d t^{\prime} .
$$


On the Fourier side, ignoring resonant terms, one sees that for $g \in L^{2}$ the term with Fourier coefficients

$$
\begin{aligned}
& \sum_{k_{1}+k_{2}+k_{3}=k} \int_{0}^{t} e^{-2 i\left(k_{1}+k_{2}\right)\left(k_{2}+k_{3}\right) t^{\prime}} \widehat{g}\left(k_{1}\right) \widehat{g}\left(k_{2}\right) \widehat{g}\left(k_{3}\right) d t^{\prime} \\
& \quad=\sum_{k_{1}+k_{2}+k_{3}=k} \frac{\widehat{g}\left(k_{1}\right) \widehat{g}\left(k_{2}\right) \widehat{g}\left(k_{3}\right)}{2\left(k_{1}+k_{2}\right)\left(k_{2}+k_{3}\right)}\left(e^{-2 i\left(k_{1}+k_{2}\right)\left(k_{2}+k_{3}\right) t}-1\right)
\end{aligned}
$$

is in $H^{\frac{1}{2}-}$ but not necessarily better.

\section{Acknowledgments}

The authors were partially supported by NSF grants DMS-1201872 (B. E.), and DMS0901222 (N. T.).

\section{References}

[1] M.V. Berry, Quantum fractals in boxes J. Phys. A: Math. Gen. 29 (1996), 6617-6629.

[2] M.V. Berry and S. Klein, Integer, fractional and fractal Talbot effects, J. Mod. Opt. 43 (1996), 2139-2164.

[3] M.V. Berry and Z.V. Lewis, On the Wierstrass-Mandelbrot fractal function, Proc. R. Soc. London A 370 (1980), 459-484.

[4] M.V. Berry, I. Marzoli and W. Schleich, Quantum carpets, carpets of light, Phys. World 14 (6) (2001), 39-44.

[5] J. Bourgain, Fourier transform restriction phenomena for certain lattice subsets and applications to non-linear evolution equations. Part I: Schrödinger equations, GAFA 3 (1993), 209-262.

[6] _ Fourier transform restriction phenomena for cer tain lattice subsets and applications to non-linear evolution equations. Part II: The KdV equation, GAFA 3 (1993), 209-262.

[7] _ Global solutions of non-linear Schrödinger equations, AMS Colloquium Publications 46, American Mathematical Society, Providence, RI, 1999.

[8] G. Chen and P.J. Olver, Numerical simulation of non-linear dispersive quantization, Discrete Contin. Dyn. Syst. 34 (3) (2014), 991-1008.

[9] _ Dispersion of discontinuous periodic waves, Proc. R. Soc. Lond. Ser. A Math. Phys. Eng. Sci. 469(2149), 20120407, 21 pp.

[10] M. Christ, Power series solution of a non-linear Schrödinger equation, in (Mathematical aspects of non-linear dispersive equations), 131-155, Ann. Math. Stud., 163, Princeton University Press, Princeton, NJ, 2007.

[11] A. Deliu and B. Jawerth, Geometrical dimension versus smoothness, Constr. Approx. 8 (1992), $211-222$

[12] M.B. Erdogan and N. Tzirakis, Global smoothing for the periodic KdV evolution, Int. Math. Res. Notices (20) (2013), 4589-4614.

[13] Smoothing and global attractors for the Zakharov system on the torus, Anal. PDE 6(3) (2013), 723-750.

[14] L. Kapitanski and I. Rodnianski, Does a quantum particle knows the time?, in 'Emerging applications of number theory, (D. Hejhal, J. Friedman, M.C. Gutzwiller, A.M. Odlyzko, eds.), IMA Volumes in Mathematics and its Applications', 109, Springer Verlag, New York, 1999, pp. $355-371$.

[15] C.E. Kenig, G. Ponce and L. Vega, A bilinear estimate with applications to the KdV equation, J. Amer. Math. Soc. 9 (1996), 573-603.

[16] P.J. Olver, Dispersive quantization, Amer. Math. Monthly 117(7) (2010), 599-610.

[17] K.I. Oskolkov, A class of I.M. Vinogradov's series and its applications in harmonic analysis, in: ('Progress in approximation theory') (Tampa, FL, 1990), Springer Ser. Comput. Math. 19, Springer, New York, 1992, pp. 353-402.

[18] L. Rayleigh, On copying diffraction-gratings, and on some phenomena connected therewith, Philos. Mag. 11 (1881), 196-205. 
[19] I. Rodnianski, Fractal solutions of the Schrödinger equation, Contemp. Math. 255 (2000), 181-187.

[20] H.F. Talbot, Facts related to optical science, No. IV, Philos. Mag. 9 (1836), 401-407.

[21] M. Taylor, The Schrödinger equation on spheres, Pacific J. Math. 209 (2003), 145-155.

[22] _ Tidbits in Harmonic Analysis, Lecture Notes, UNC, 1998.

[23] H. Triebel, Theory of function spaces, Birkhäuser, Basel, 1983.

[24] Y. Zhang, J. Wen, S.N. Zhu and M. Xiao, Nonlinear Talbot effect, Phys. Rev. Lett. 104 (2010), 183901.

Department of Mathematics, University of Illinois Urbana-Champaign, 1409 W. Green Street URBAnA, IL 61801, USA

E-mail address: berdogan@math.uiuc.edu

E-mail address: tzirakis@math.uiuc.edu 